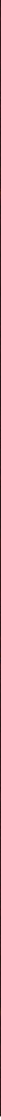

Credit: Gerry Shaw/Wikimedia Commons

W the human brain remains one of our greatest scientific mysteries, as well as one of our largest technical challenges. Despite recent advances in our understanding of the brain, the underlying causes of most neurological and psychiatric disorders remain largely uncharacterized. Many researchers think that developing more effective treatments for these conditions will require an arsenal of tools and methods aimed at deciphering brain function in health and disease.

To address this need, former President Barack Obama launched the BRAIN (Brain Research through Advancing Innovative Neurotechnologies) Initiative in April 2013. The project will scale from atoms to thoughts and behavior, linking what is known about single cells and subcellular activity with whole-brain function.

To organize such a large-scale effort, the National Institutes of Health $(\mathrm{NIH})$ convened a working group to develop a rigorous plan for achieving those goals. The working group's BRAIN 2025 report, released in 2014, outlines how the initiative should progress over a 10-year period beginning in fiscal year 2016, with a primary focus on technology development, followed by a shift in later years to integrating these technologies in an effort to make new discoveries.

\section{Cell census}

"One limitation is that we so far lack a comprehensive parts list of all the neurons that comprise the brain and a wiring diagram of how they connect to each other," notes John Ngai from the University of California, Berkeley. "How do these different cell types work together to serve the functioning of this exquisitely complex biological computer?"

Supported through the BRAIN Initiative, Ngai and his team are now working to classify cortical neurons using single-cell transcriptomics. To understand what makes a neuron distinct, Ngai's group is trying to pinpoint the specific genes responsible for differentiating a cell into various subtypes and then testing whether each subtype has a unique function by tagging them with labels.

"We can use this information to identify novel cell types that previously eluded detection, simply because we did not have knowledge of markers that otherwise could be used to identify them," Ngai says. "Ultimately, we can use single-cell transcriptomics as a foundation to acquire information about a cell's location in the brain, morphology-including its connectivity with other neurons in the brain-and physiology, which together should provide all the information needed to identify, classify, and characterize every cell type in the mammalian brain."

As they reported in the June 2017 issue of Cell Stem Cell, Ngai's team developed an integrated approach based on in silico analysis of single-cell RNA sequencing data and in vivo lineage tracing-a technique for permanently labeling the descendants of a targeted cell. Using this method, they illuminated the cell fate potentials of individual olfactory stem cells and the locations of branchpoints in the olfactory lineage trajectory at a level of detail not possible by either in vivo lineage tracing or single-cell RNA sequencing alone [1].

"For the first time, we are now able to identify cell types and cell states within a heterogeneous population of cells without any a priori knowledge of what distinguishes one type or state from another," Ngai says. "We literally caught cells in the act of choosing one cell fate over another and identified some of the genes driving such decisions."

This combined analysis identified trajectories that produce major cell types in the olfactory epithelium, and it serves as a model for elucidating complex lineage trajectories in other stem cell niches. According to the authors, detailed lineage trajectory maps are necessary for identifying the regulatory networks that govern the cell fate transitions underlying tissue maintenance and regeneration, and are essential in designing strategies to manipulate cells for therapeutic applications. 


\section{Large-scale, single-cell analysis}

On the opposite coast at Harvard University, Joshua Sanes and his team are also using single-cell transcriptomics in their work, focusing on new methods of genetic screening to catalog and distinguish different cell types across species and brain regions. "Our BRAIN grant was designed to compare various single-cell methods, using the retina as the main model, with the aim of then using the winner to generate a full cell atlas," explains Sanes.

In the May 2015 issue of Cell, Sanes and his colleagues described Drop-seq, a method for quickly profiling thousands of individual cells by separating them into nanoliter-sized aqueous droplets, associating a different barcode with each cell's RNAs, and then sequencing them all together [2]. Drop-seq analyzes mRNA transcripts from thousands of individual cells simultaneously while remembering each transcript's cell of origin. Using Dropseq, Sanes' team analyzed transcriptomes from 44,808 mouse retinal cells and identified 39 transcriptionally distinct cell populations, creating a molecular atlas of gene expression for known retinal cell classes and novel candidate cell subtypes.

"Drop-seq allowed transcriptomic analysis of tens of thousands of cells for the first time, by bringing the monetary and labor costs down by orders of magnitude," Sanes says. "In fact, this paper may have reported as many single-cell transcriptomes as had been reported in all previous studies put together. The methodological innovation wasn't just hardware though-improved bioinformatics methods were needed to process and comprehend the massive data sets."

In a follow-up study published in August 2016 in Cell, Sanes and his team developed and applied an integrated strategy for building a comprehensive validated atlas of cell types [3]. Using Drop-seq, along with optimized computational methods, the researchers studied a diverse class of neurons: mouse retinal bipolar cells. "Even though neuronal types in the retina have been studied for nearly 150 years, our work has identified multiple novel types,"
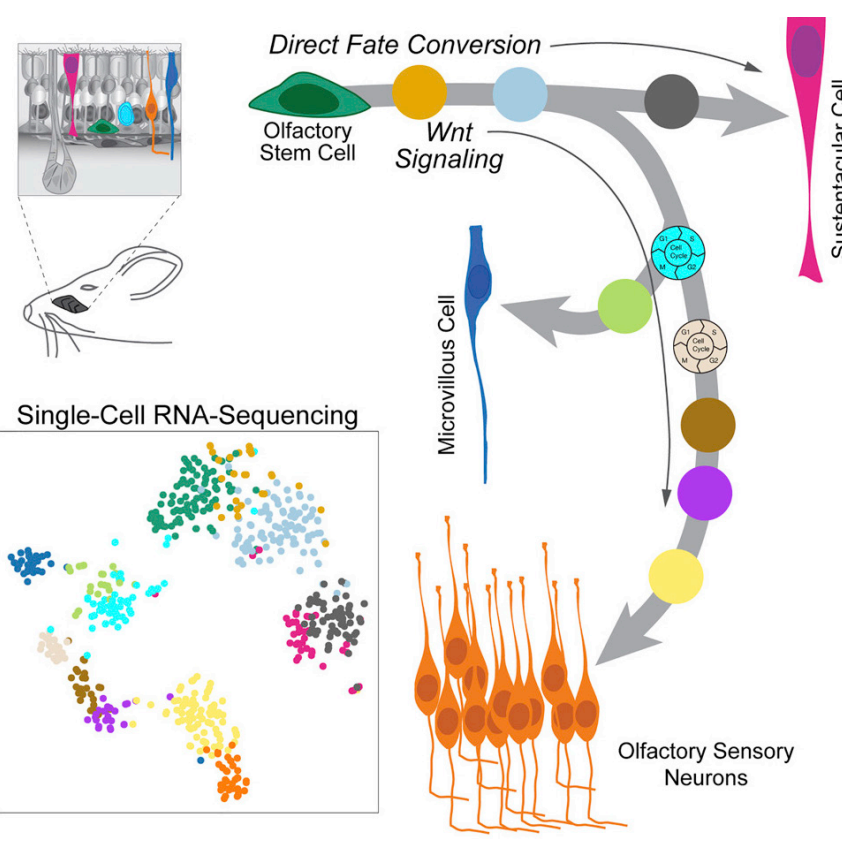

John Ngai and his colleagues at the University of California, Berkeley used in silico analysis of RNA-seq data along with lineage tracing to explore cell fate in olfactory stem cells. Courtesy of J. Ngai.

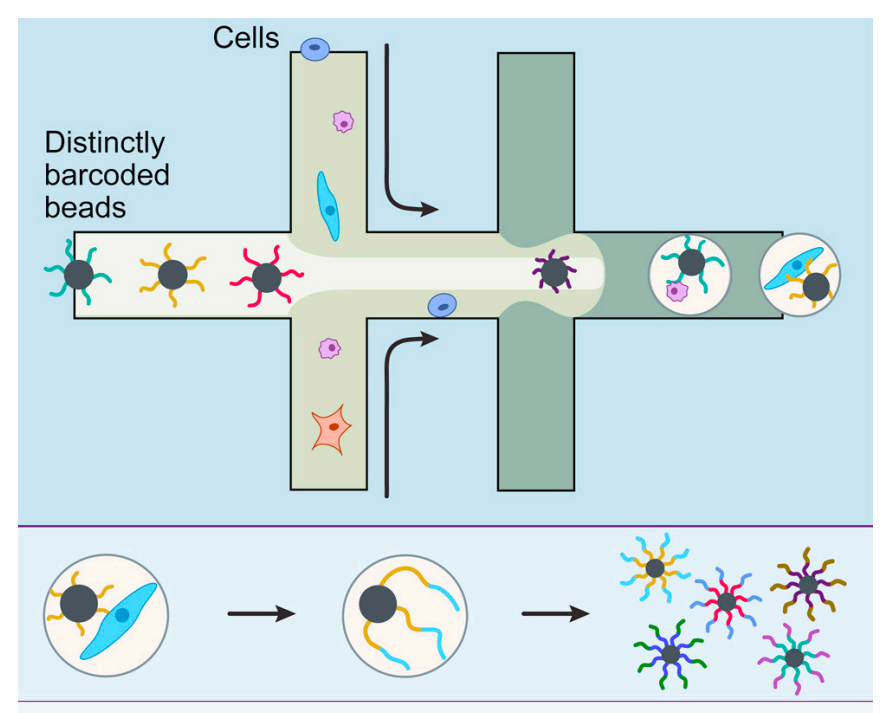

Schematic representation of the Drop-seq single-cell analysis technique developed by Josh Sanes and colleagues. Courtesy of J. Sanes.

says first author Karthik Shekhar, a postdoctoral associate at the Broad Institute of MIT and Harvard.

From a population of approximately 25,000 bipolar cells, the team derived molecular classifications for 15 types, including all cell types observed previously plus 2 novel cell types. "We are close to completing the atlas of neuronal types for the retina, which will be the first of its kind in the mammalian brain," says Shekhar.

According to the authors, their work provides a systematic methodology for achieving comprehensive molecular classification of neurons, identifies novel neuronal types, and uncovers transcriptional differences that distinguish types within a class. And with advances in multiplexing technologies and decreasing sequencing costs, future studies will be able to profile even larger numbers of cells at even greater depth.

"I believe that inability to comprehensively classify neurons is a major bottleneck to understanding neural function and dysfunction throughout the brain," Sanes asserts. "I hope that success in the retina would allow us to optimize a pipeline that could then be applied to less tractable regions such as the cerebral cortex."

According to Sanes, these efforts are essential for finding out how circuits malfunction in brain diseases. "For example, some disorders may seem especially mysterious because they affect just a small number of the many types of neurons or glia in the brain, making it nearly impossible to track down the needle in the haystack," he says. "As we classify neurons, and develop ways to mark and even purify them, we will be able to ask whether there are particularly vulnerable types." In fact, the work done by Sanes' team on the retina has already exposed some cell types that are more resilient to nerve injury than others.

\section{References}

1. Fletcher R.B. et al. 2017. Deconstructing olfactory stem cell trajectories at single-cell resolution. Cell Stem Cell. 20:817-830.e8.

2. Macosko E.Z. et al. 2015. Highly parallel genome-wide expression profiling of individual cells using nanoliter droplets. Cell. 161:1202-1214.

3. Shekhar K. et al. 2016. Comprehensive classification of retinal bipolar neurons by single-cell transcriptomics. Cell. 166:1308-1323.e30.

Written by Janelle Weaver. 迎

BioTechniques 63:158-159 (October 2017) doi: 10.2144/000114600 